\title{
Quantitative analysis of metal artifact reduction in total hip arthroplasty using virtual monochromatic imaging and orthopedic metal artifact reduction, a phantom study
}

\author{
Mark Selles ${ }^{1 *}$ (D) Vera H. Stuivenberg ${ }^{1}$, Ruud H. H. Wellenberg ${ }^{2}$, Loes van de Riet ${ }^{1,4}$, Ingrid M. Nijholt ${ }^{1}$,
} Jochen A. C. van Osch ${ }^{3}$, Robbert W. van Hamersvelt ${ }^{4}$, Tim Leiner ${ }^{4}$ and Martijn F. Boomsma ${ }^{1}$

\begin{abstract}
Objective: To quantify metal artifact reduction using $130 \mathrm{keV}$ virtual monochromatic imaging (VMI) with and without orthopedic metal artifact reduction (O-MAR) in total hip arthroplasty.

Methods: Conventional polychromatic images and $130 \mathrm{keVVMI}$ of a phantom with pellets representing bone with unilateral or bilateral prostheses were reconstructed with and without O-MAR on a dual-layer CT. Pellets were categorized as unaffected, mildly affected and severely affected.

Results: When 130 keV VMI with O-MAR was compared to conventional imaging with O-MAR, a relative metal artifact reduction in CT values, contrast-to-noise (CNR), signal-to-noise (SNR) and noise in mildly affected pellets (67\%, 74\%, $48 \%, 68 \%$, respectively; $p<0.05)$ was observed but no significant relative metal artifact reduction in severely affected pellets. Comparison between $130 \mathrm{keV} \mathrm{VMI} \mathrm{without} \mathrm{O-MAR} \mathrm{and} \mathrm{conventional} \mathrm{imaging} \mathrm{with} \mathrm{O-MAR} \mathrm{showed} \mathrm{relative}$ metal artifact reduction in CT values, CNR, SNR and noise in mildly affected pellets ( $92 \%, 72 \%, 38 \%, 51 \%$, respectively; $p<0.05)$ but negative relative metal artifact reduction in CT values and noise in severely affected pellets $(-331 \%$ and $-223 \%$, respectively; $p<0.05$ ), indicating aggravation of metal artifacts.
\end{abstract}

Conclusion: Overall, VMI of $130 \mathrm{keV}$ with O-MAR provided the strongest metal artifact reduction.

Keywords: Arthroplasty, Replacement, Hip, CT, Dual energy

\section{Key points}

- $130 \mathrm{keV}$ VMI with O-MAR results in strongest metal artifact reduction.

- Only mild metal artifacts are reduced by $130 \mathrm{keV}$ VMI without O-MAR.

- Severe metal artifacts are already reduced by conventional imaging with O-MAR.

\footnotetext{
${ }^{*}$ Correspondence: m.selles@isala.nl

${ }^{1}$ Department of Radiology, Isala, 8025 AB Zwolle, The Netherlands

Full list of author information is available at the end of the article
}

\section{Background}

Computed tomography (CT) is commonly used as imaging modality during postoperative follow-up after total hip arthroplasties (THA) [1]. CT is a relatively inexpensive, readily available modality and it is very effective in visualizing bone defects [2]. However, imaging of the pelvis in patients with large metal hip prostheses remains one of the biggest challenges in CT imaging since metallic prosthetic components lead to metal artifacts that make the assessment of surrounding bone and soft tissues difficult. Metal artifacts also hamper the detection of pathological capsular reactions of THA components [2]. 
Metal artifacts arise from beam hardening, scatter and photon starvation [2].

Several methods are available to reduce these metal artifacts. This includes basic steps such as increasing tube voltage and proper positioning of the patient. However, increasing tube voltage results in a higher required dose. More advanced methods include the use of model-based image reconstruction, iterative image reconstruction and metal artifact reduction (MAR) software [2, 3]. The artifact reduction ability of such software in conventional CT imaging of THA can be further increased by the addition of iterative model-based reconstruction (IMR) [1]. Another option to reduce artifacts is the use of dual-energy CT (DECT) with reconstruction of virtual monochromatic images at high energy levels. Prior work by Wellenberg and co-workers showed that the optimal monochromatic energy depends on the material of the prostheses. In general, virtual monochromatic imaging (VMI) at $130 \mathrm{keV}$ was the optimal monochromatic energy level for metal artifact reduction in THA [4]. Several patient studies showed that the combination of MAR software and virtual monochromatic dual-energy CT imaging reduces metal artifacts even more effectively than one technique alone [5-7].

However, quantification of metal artifacts in patient studies is limited by anatomical differences between patients. By using a THA phantom, scans without hip prostheses can serve as a direct reference for scans with hip prostheses. This allows systematic assessment of metal artifacts and quantitative analysis which is not possible in patients with THA. Hitherto, a thorough, quantitative analysis of metal artifact reduction by combined use of DECT and orthopedic metal artifact reduction (O-MAR) in a THA phantom has not been performed. Hence, the aim of this study was to quantitatively compare the extent to which metal artifacts in a THA phantom can be reduced by $130 \mathrm{keV}$ VMI with O-MAR, $130 \mathrm{keV}$ VMI without O-MAR, conventional polychromatic imaging with O-MAR and conventional imaging without O-MAR.

\section{Methods \\ Phantom}

The THA phantom was made of polymethyl methacrylate (PMMA) with the following dimensions: $320 \mathrm{~mm}$ wide, $130 \mathrm{~mm}$ high and $290 \mathrm{~mm}$ deep. The phantom contained 18 cylindrical pellets with a height and diameter of $10 \mathrm{~mm}$ (Fig. 1). These hydroxyapatite/calcium carbonate pellets all represented the same modular bone density and CT values of bone. The nine pellets on each side of the phantom were located at the acetabulum zone (DeLee and Charnley zones, pellets 1 and 2) and the most relevant radiological zones of the femur (the Gruen zones, pellets 3-9) (Fig. 2) [8, 9]. The phantom was filled with water, and air bubbles were removed.

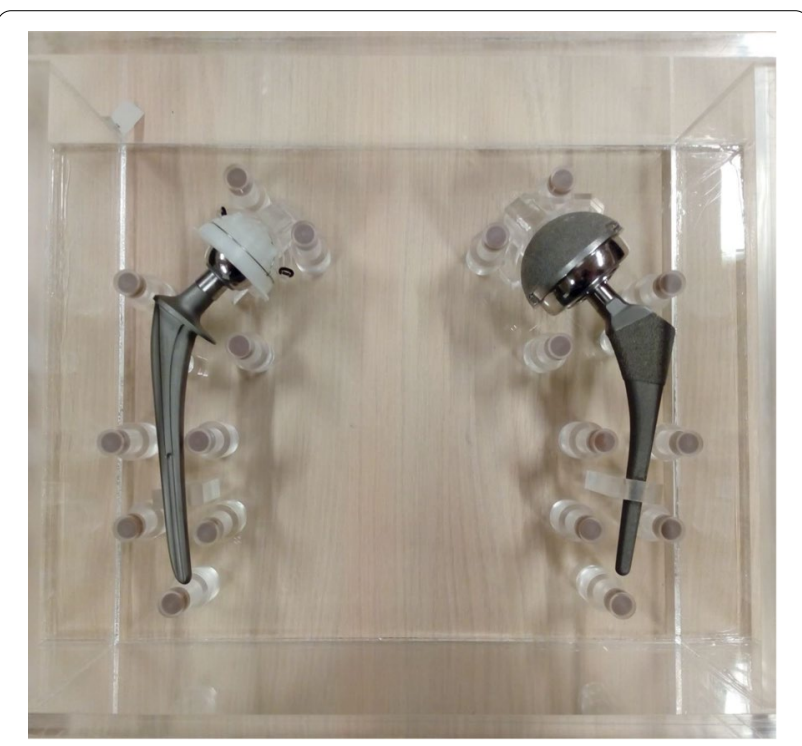

Fig. 1 Image of the phantom containing bilateral prostheses at the right (cup: UHMWPE, head: ZTA, stem: CoCrMo) and left (cup: CoCrMo, head: CoCrMo, stem: TiAIV). UHMWPE ultrahigh molecular weight erthylene, ZTA Zirconia toughened aluminum, TiAIV titaniumaluminum-vanadium, CoCrMo cobalt-chrome-molybdenum

\section{Image acquisition and reconstruction}

Polychromatic and virtual monochromatic CT images were acquired on a Philips IQon 128 slice dual-layer detector CT scanner. The scan protocol was similar to clinical practice with regard to clinical evaluation of hip prostheses, using a standard CT dose index (CTDI) of $20 \mathrm{mGy}$ at $140-\mathrm{kVp}$ (Table 1). The phantom was imaged with either a unilateral or bilateral total hip prosthesis (Fig. 2). Acquisitions without prostheses served as a reference. The materials used in the phantom with unilateral prosthesis were ultrahigh molecular weight erthylene (UHMWPE) as prosthesis cup, Zirconia toughened aluminum (ZTA) as prosthesis head and titanium-aluminum-vanadium (TiAlV) as prosthesis stem. The materials used in the phantom with bilateral prostheses were UHMWPE (cup), ZTA (head) and CoCrMo (stem) for the right prosthesis and CoCrMo (cup), CoCrMo (head) and TiAlV (stem) for the left prosthesis. The materials of the head and stem and the UHMWPE cup are the commonly used for THA procedures in The Netherlands [10]. Although not commonly used, we chose to include a CoCrMo cup which aggravates metal artifacts as our aim was to explore to which extent metal artifacts can be reduced by different reconstructions.

Figure 3 shows the design of this study. Polychromatic CT images acquired at $140 \mathrm{kVp}$ were reconstructed using IMR level 1 and further referred to as 'conventional'. Virtual monochromatic images (VMI) at $130 \mathrm{keV}$ were extracted, since this $\mathrm{keV}$ resulted in 


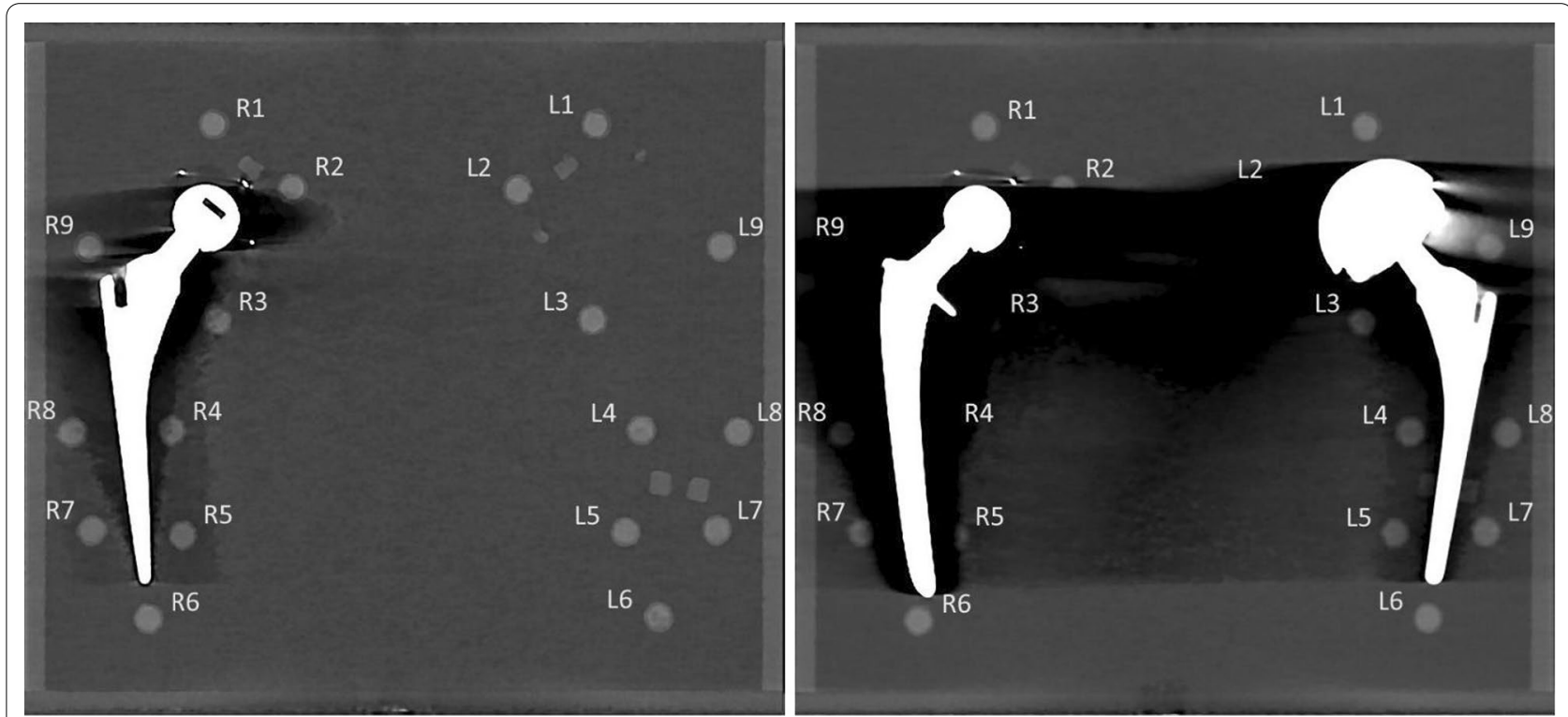

Fig. $2140 \mathrm{kVp}$ polychromatic images of the phantom. Left image: phantom with unilateral prosthesis at the right (cup: UHMWPE, head: ZTA, stem: TiAIV). Right image: phantom with bilateral prostheses at the right (cup: UHMWPE, head: ZTA, stem: CoCrMo) and left (cup: CoCrMo, head: CoCrMo, stem: TiAIV). UHMWPE ultrahigh molecular weight erthylene, ZTA Zirconia toughene aluminum, TiAlV titanium-aluminum-vanadium, CoCrMo cobalt-chrome-molybdenum

Table 1 Scan protocol

\begin{tabular}{ll}
\hline & $\begin{array}{l}\text { Philips iQon 128 slice } \\
\text { dual-layer detector } \\
\text { CT }\end{array}$ \\
\hline Energy & $140 \mathrm{kVp}$ \\
Collimation & $64 \times 0.625 \mathrm{~mm}$ \\
FOV & $330 \mathrm{~mm}$ \\
Pitch & 0.392 \\
Matrix & $512 \times 512$ \\
Rotation time & $0.75 \mathrm{~s}$ \\
Dose (CTDI) & $20 \mathrm{mGy}$ \\
Filter & IMR: sharp plus \\
& Spectral level: sharp (C) \\
Increment & 0.46 \\
Slice thickness & $0.9 \mathrm{~mm}$ \\
\hline
\end{tabular}

optimal CNR in a similar previous study [4]. Both conventional images and $130 \mathrm{keV}$ virtual monochromatic images were reconstructed with and without O-MAR (Philips Healthcare, Best, The Netherlands).

\section{Analysis of image quality}

For all obtained images, the coronal slice located in the middle of the prosthesis was extracted and used for quantitative analysis in Image (1.48v). The images were analyzed by manually drawing nine regions of interest (ROIs) placed in the pellets at the left (L1-L9), nine ROIs placed in the pellets at the right (R1-R9) and one ROI in a homogeneous section of water which was unexposed to metal artifacts (Fig. 4). The diameter of the ROIs was $6.75 \mathrm{~mm}$ or 15 pixels to limit partial volume effects. To allow systematic analysis of the images, a standardized template of ROIs was used in ImageJ. The standardized template was used to measure CT numbers in Hounsfield units (HU) and the standard deviation in $\mathrm{HU}$ as a measure of noise. Signal-to-noise ratio (SNR) was calculated by dividing the $\mathrm{HU}$ by the noise of the pellets. Contrastto-noise ratio (CNR) was calculated by dividing the difference in CT values between the pellet and background by the mean of the noise of the pellet and background noise.

Pellets were categorized as unaffected, mildly affected or severely affected based on the mean HU of the pellet in the unilateral and bilateral conventional images without O-MAR (Fig. 4). We chose to categorize the pellets to explore metal artifact reduction of mild and severe artifacts. Pellets with mean $\mathrm{CT}$ value $<0 \mathrm{HU}$ were categorized as severely affected, pellets with mean $\mathrm{CT}$ value $>0$ $\mathrm{HU}$ and $\mathrm{CT}$ value $<235 \mathrm{HU}$ were categorized as mildly affected and pellets with CT value $\geq 235$ HU were categorized as unaffected (Fig. 5). The cutoff value of $235 \mathrm{HU}$ was determined using the conventional reference image without O-MAR. CT values $<235 \mathrm{HU}$ were found to deviate more than the standard deviation from reference values and were therefore considered affected. 


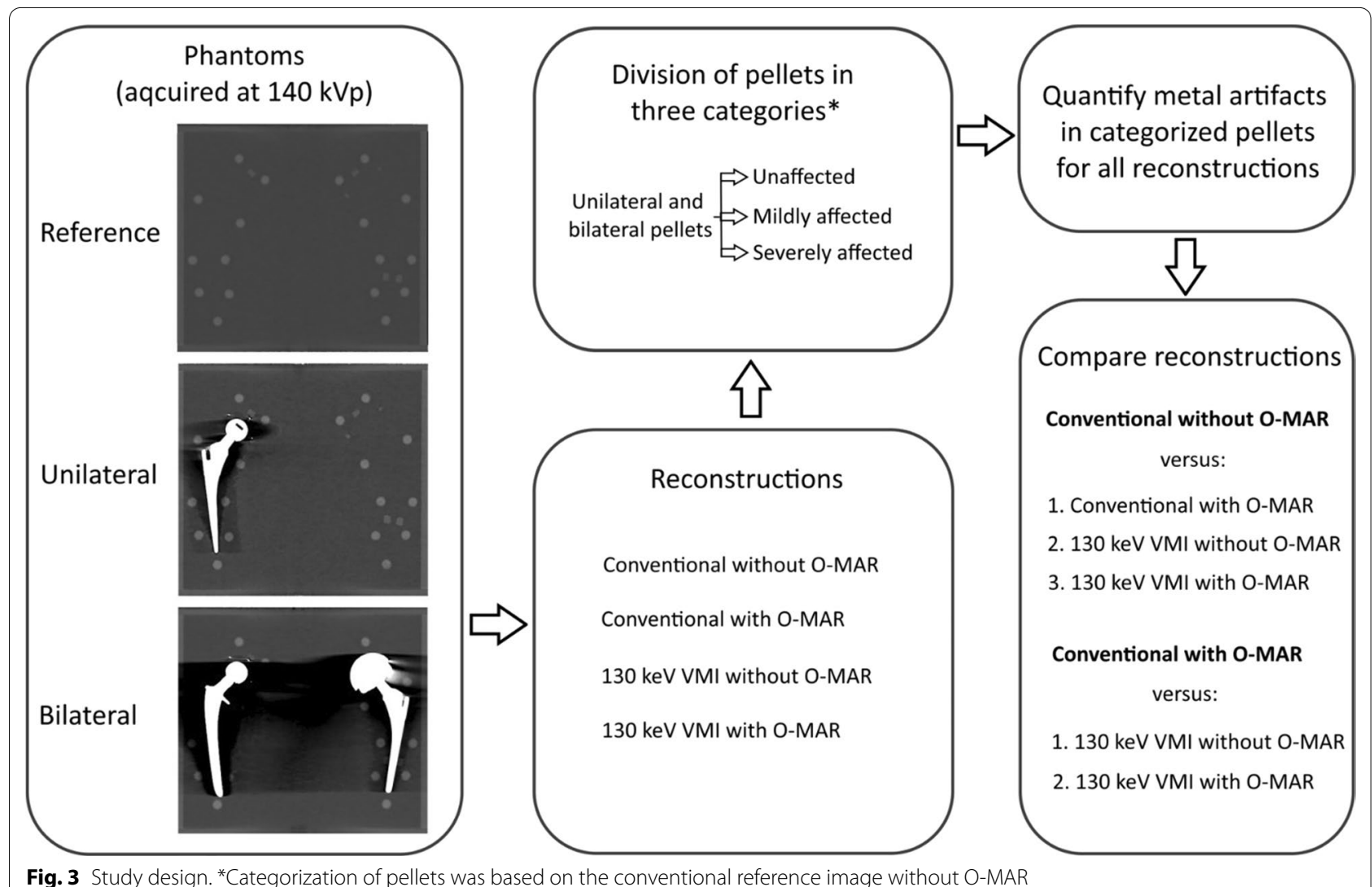

Fig. 3 Study design. ${ }^{*}$ Categorization of pellets was based on the conventional reference image without O-MAR
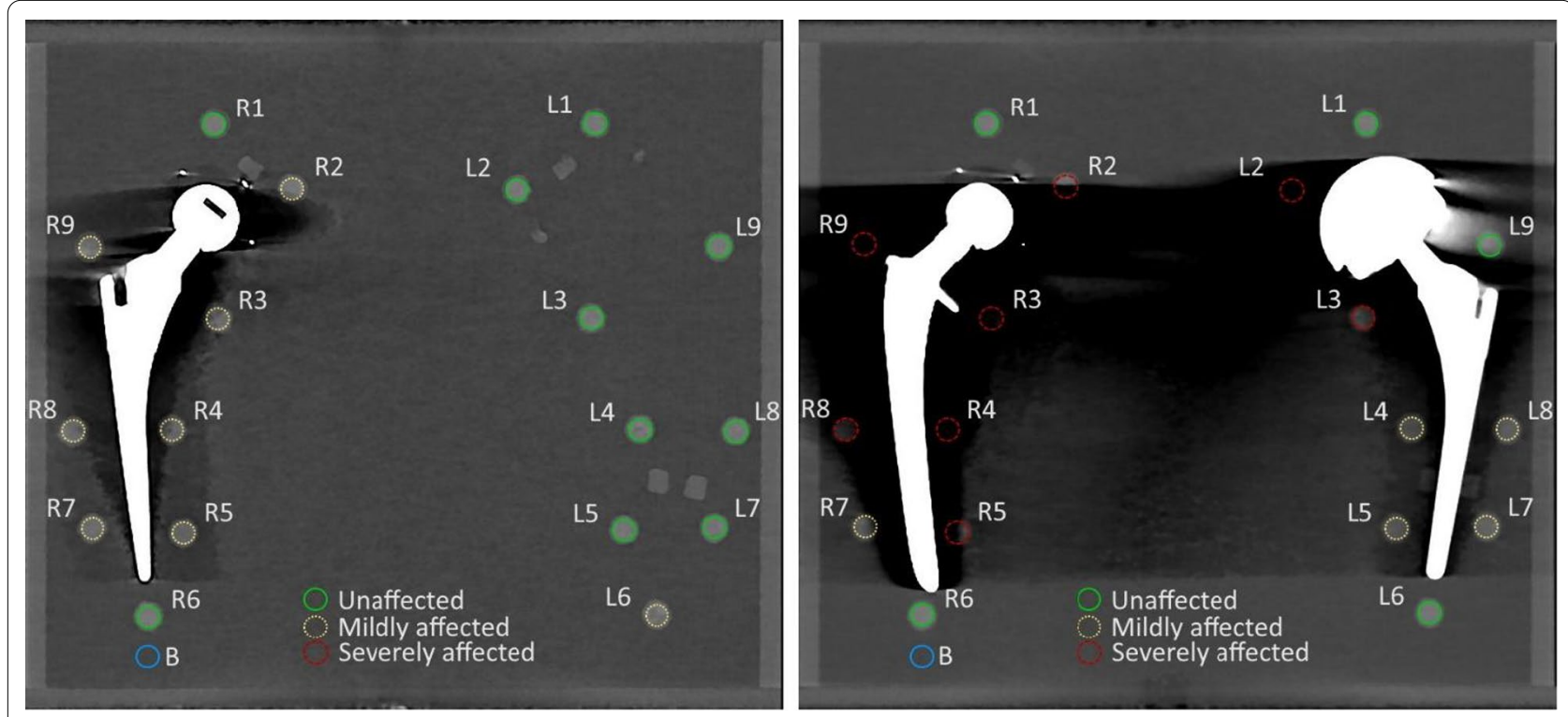

Fig. 4: $140 \mathrm{kVp}$ conventional images without O-MAR with a unilateral prosthesis (left) and bilateral prostheses (right). Pellets were categorized as unaffected (green), mildly affected (yellow) and severely affected (red) pellets. The background region of interest (blue) was used to calculate contrast-to-noise ratio 


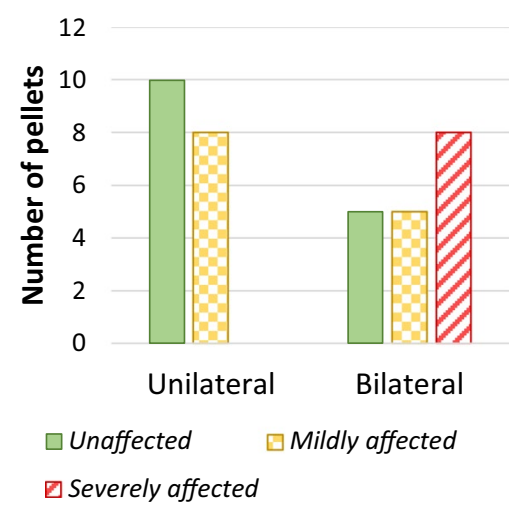

Fig. 5 Number of pellets classified as unaffected, mildly affected or severely affected for unilateral and bilateral prostheses

\section{Quantification of metal artifact reduction}

The absolute difference in mean CT values, SNR, CNR or noise between the images with prostheses and reference images (without prostheses) was calculated for mildly and severely affected artifacts as a measure of metal artifact reduction. The difference was normalized as calculated by

$$
\Delta V_{\text {reconstruction }}=\left|1-\frac{V_{\text {with prostheses }}}{V_{\text {no prostheses }}}\right|_{\text {reconstruction }}
$$

where $V$ is either CT values, SNR, CNR or noise. Normalization was applied to allow comparison between the VMI and conventional images as the CT values on the VMI are notably lower due to the high virtual monochromatic energy.

The calculated differences for CT values, SNR, CNR and noise were compared for the reconstructions for mildly and severely affected artifacts to define the relative metal artifact reduction as follows:

Relative metal artifact reduction(\%)

$$
=\left(1-\left(\frac{\Delta V_{\text {reconstruction }}}{\Delta V_{\text {standard reconstruction }}}\right)\right) * 100 \%
$$

where $\Delta V$ is calculated as shown in Eq. 1. Conventional imaging without $\mathrm{O}-\mathrm{MAR}$ and conventional imaging with O-MAR were used as standard reconstructions in Eq. 2 because these images are commonly used in clinical practice. Relative metal artifact reduction was calculated to compare reconstructions. VMI of $130 \mathrm{keV}$ with O-MAR, VMI of $130 \mathrm{keV}$ without O-MAR and conventional imaging with $\mathrm{O}-\mathrm{MAR}$ were compared to conventional imaging without O-MAR. VMI of $130 \mathrm{keV}$ with O-MAR and VMI of $130 \mathrm{keV}$ without O-MAR were compared to conventional imaging with O-MAR (Fig. 3).

\section{Statistical analysis}

Wilcoxon signed-ranks tests were used to test for differences in metal artifact reduction between the reconstructions. A two-sided alpha of 5\% was used as significance level. IBM SPSS software (version 23) was used for analysis.

\section{Results}

Image quality parameters of conventional and VMI without O-MAR were different without insertion of prostheses (Table 2). Reconstructions with O-MAR resulted in the exact same values for these parameters as O-MAR does not change CT values when no metal is present.

Conventional images of the phantom with bilateral protheses showed more streak artifacts compared to conventional images of the phantom with unilateral prosthesis. Conventional imaging with O-MAR resulted in a decrease of streak artifacts for both unilateral and bilateral images. VMI of $130 \mathrm{keV}$ without O-MAR resulted in a decrease of streak artifacts, in particular for the unilateral prosthesis. However, bright streak artifacts were introduced on the $130 \mathrm{keV}$ VMI. VMI of $130 \mathrm{keV}$ with O-MAR reduced dark streak artifacts without introducing bright artifacts (Fig. 6).

The highest CT values, CNR and SNR were found in conventional images with O-MAR for both mildly as severely affected pellets. The difference between both mildly and severely affected pellets and reference in CT

Table 2 Mean image quality parameters for conventional $140 \mathrm{kVp}$ and $130 \mathrm{keV}$ virtual monochromatic imaging with and without

\begin{tabular}{|c|c|c|c|c|}
\hline & CT values $(\mathrm{HU})$ & CNR & SNR & Noise (HU) \\
\hline Conventional & $248.4 \pm 4.1$ & $37.3 \pm 2.8$ & $36.5 \pm 5.2$ & $6.9 \pm 1.0$ \\
\hline Conventional with O-MAR & $248.4 \pm 4.1$ & $37.3 \pm 2.8$ & $36.5 \pm 5.2$ & $6.9 \pm 1.0$ \\
\hline 130 keV VMI & $150.4 \pm 4.5$ & $18.0 \pm 1.5$ & $19.3 \pm 3.2$ & $8.0 \pm 1.2$ \\
\hline 130 keV VMI with O-MAR & $150.4 \pm 4.5$ & $18.0 \pm 1.5$ & $19.3 \pm 3.2$ & $8.0 \pm 1.2$ \\
\hline
\end{tabular}
$\mathrm{O}-\mathrm{MAR}$, without insertion of prostheses. Values are the mean of all 18 pellets \pm standard deviation 


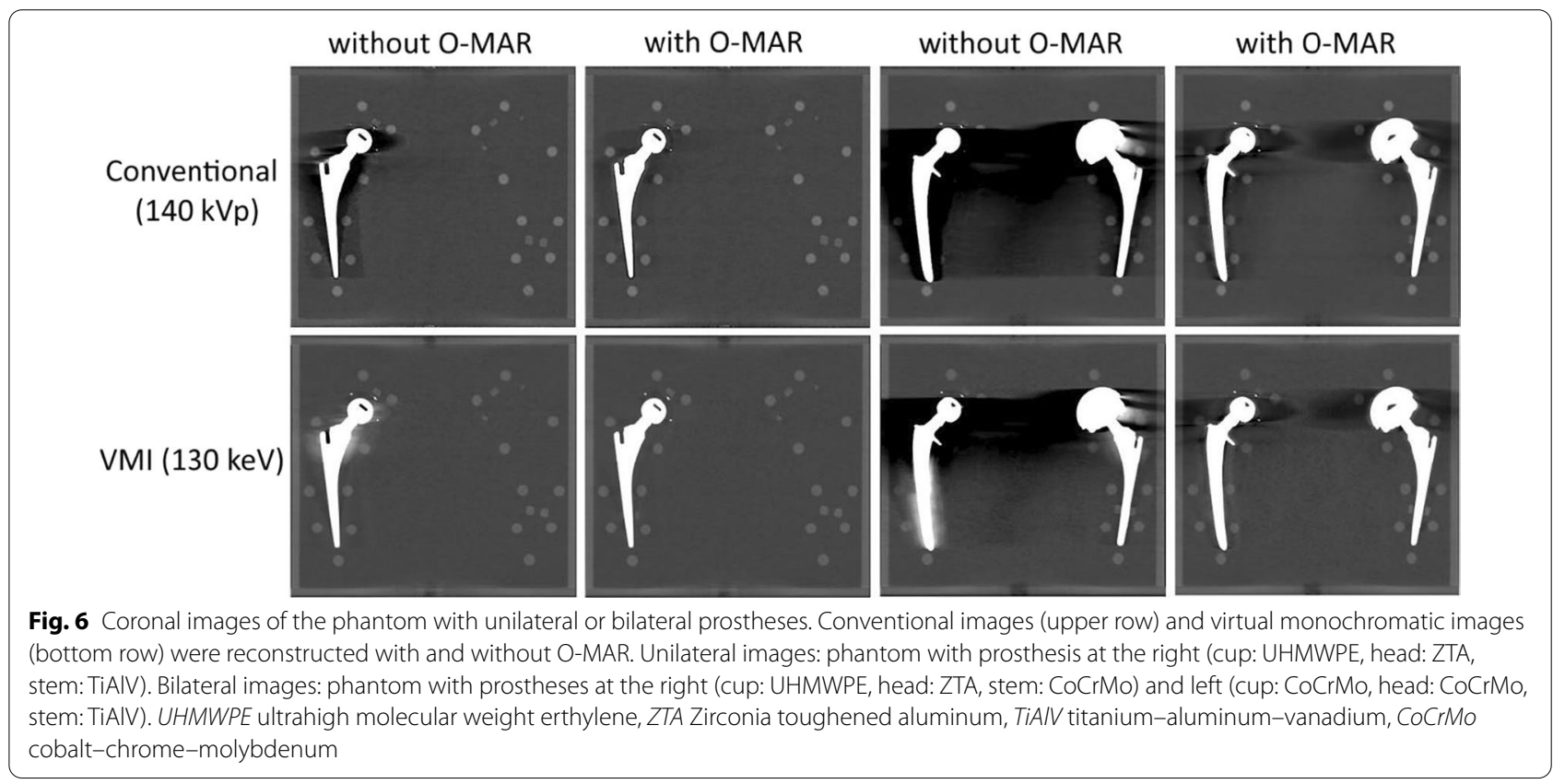

values, CNR and SNR were lowest for $130 \mathrm{keV}$ images with O-MAR. Also, the lowest noise was measured on the $130 \mathrm{keV}$ images with O-MAR. VMI of $130 \mathrm{keV}$ without O-MAR showed similar results to $130 \mathrm{keV}$ images with O-MAR in CT values, CNR, SNR and noise for mildly affected pellets. However, CT values, CNR, SNR and noise were much lower and noise was much higher on the $130 \mathrm{keV}$ images without O-MAR in comparison to $130 \mathrm{keV}$ images with O-MAR for severely affected pellets (Fig. 7, see Additional file 1 for data of all individual pellets).

Conventional imaging with O-MAR resulted in significant metal artifact reduction in CT values, CNR, SNR and noise for mildly affected pellets $(45 \%, 34 \%, 33 \%$ and $71 \%$, respectively) and severely affected pellets (77\%, $36 \%, 26 \%$ and $84 \%$, respectively) in comparison to conventional imaging without O-MAR (Table 3). VMI of $130 \mathrm{keV}$ without O-MAR resulted in significant metal artifact reduction for mildly affected pellets in CT values, CNR, SNR and noise $(95 \%, 81 \%, 58 \%$ and $86 \%$, respectively) and a significant metal artifact reduction of $47 \%$ in noise in severely affected artifacts in comparison to conventional imaging without O-MAR (Table 3). VMI of $130 \mathrm{keV}$ with O-MAR also resulted in significant metal artifact reduction in CT values, CNR, SNR and noise for mildly affected pellets $(81 \%, 83 \%, 66 \%$ and $91 \%$, respectively) and severely affected pellets $(79 \%, 46 \%, 32 \%$ and $90 \%$, respectively) in comparison to conventional imaging without O-MAR (Table 3).

VMI of $130 \mathrm{keV}$ without O-MAR resulted in significant metal artifact reduction in CT values, CNR and SNR in mildly affected pellets (92\%, $72 \%$ and $38 \%$, respectively) in comparison to conventional imaging with O-MAR but resulted in a significant decrease in metal artifact reduction in HU, SNR and noise in severely affected pellets ( $-331 \%,-202 \%$ and $-223 \%$, respectively; Table 4$)$. It should be noted that a negative metal artifact reduction indicates aggravation of the artifacts. VMI of $130 \mathrm{keV}$ with O-MAR resulted in significant metal artifact reduction in CT values, CNR, SNR and noise for mildly (67\%, $74 \%, 48 \%$ and $68 \%$, respectively) in comparison to conventional imaging with O-MAR (Table 4).

\section{Discussion}

In this study, we quantified metal artifact reduction in a THA phantom using high energy VMI with and without O-MAR in comparison to conventional imaging with and without O-MAR. We found that $130 \mathrm{keV}$ VMI with $\mathrm{O}-\mathrm{MAR}$ resulted in the strongest metal artifact reduction of mild and severe metal artifacts.

A tube voltage of $140 \mathrm{kVp}$ is often used to reduce metal artifacts and results in lower CT number inaccuracies, lower noise and higher SNR and CNR [11] in comparison to $120 \mathrm{kVp}$. However, increasing tube voltage may result in only a slight reduction of metal artifacts [1217]. Moreover, a higher tube voltage leads to a higher required dose whereas extraction of $\mathrm{VMI}$ and application of O-MAR are not leading to a higher required dose.

In previous THA phantom studies, it was already reported that the use of O-MAR in conventional imaging reduces metal artifacts $[1,2,18-21]$. Our results confirmed the value of O-MAR in conventional imaging for 


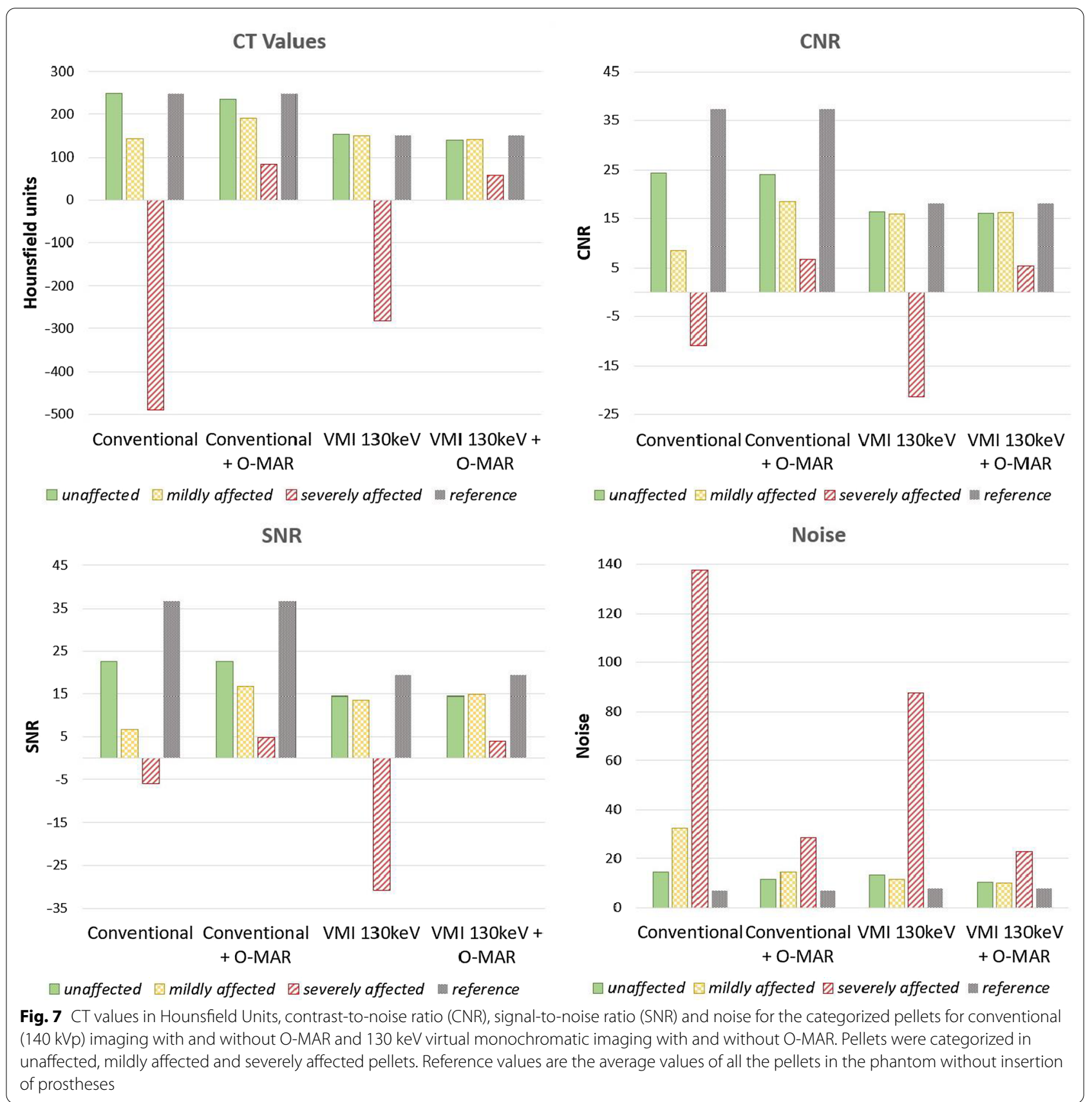

metal artifact reduction. Although $130 \mathrm{keV}$ VMI without O-MAR showed stronger metal artifact reduction for mildly affected pellets than conventional imaging with O-MAR, metal artifact reduction for severely affected pellets worsened in comparison to conventional imaging with O-MAR in our study. Besides, the metal artifact reduction in CT values of $92 \%$ when comparing $130 \mathrm{keV}$ VMI without O-MAR and conventional imaging with O-MAR is probably heightened by the bright streak artifacts visible on the $130 \mathrm{keV}$ VMI without O-MAR. Our finding that $130 \mathrm{keV}$ VMI in only mildly affected pellets showed metal artifact reduction in comparison to conventional imaging may be explained by reduction of metal artifacts caused by beam hardening. VMI of $130 \mathrm{keV}$ is less prone to beam hardening artifacts, which are dominant in mildly affected pellets, decreasing metal artifacts.

Severely affected pellets were only present in the images of the phantom with bilateral prostheses where photon starvation is dominant over beam hardening. 
Table 3 Relative metal artifact reduction in CT values (HU), contrast-to-noise ratio, signal-to-noise ratio and noise in comparison to conventional (140kVp) images without O-MAR

\begin{tabular}{|c|c|c|c|c|c|c|c|c|}
\hline & Metal artif & eduction (\% & ildly affecte & Ilets & Metal artifact & eduction ( $\%$ & verely affec & pellets \\
\hline & HU & CNR & SNR & Noise & $\mathrm{HU}$ & CNR & SNR & Noise \\
\hline $\begin{array}{l}\text { Conven- } \\
\text { tional + O-MAR }\end{array}$ & $\begin{array}{l}45 \% \\
(p=0.006)\end{array}$ & $\begin{array}{l}34 \% \\
(p=0.002)\end{array}$ & $\begin{array}{l}33 \% \\
(p=0.002)\end{array}$ & $\begin{array}{l}71 \% \\
(p=0.002)\end{array}$ & $\begin{array}{l}77 \% \\
(p=0.012)\end{array}$ & $\begin{array}{l}36 \% \\
(p=0.012)\end{array}$ & $\begin{array}{l}26 \% \\
(p=0.012)\end{array}$ & $84 \%(p=0.012)$ \\
\hline 130 keV VMI & $\begin{array}{l}95 \% \\
(p=0.003)\end{array}$ & $\begin{array}{l}81 \% \\
(p=0.001)\end{array}$ & $\begin{array}{l}58 \% \\
(p=0.001)\end{array}$ & $\begin{array}{l}86 \% \\
(p=0.001)\end{array}$ & $3 \%(p=0.779)$ & $\begin{array}{l}-69 \% \\
(p=0.889)\end{array}$ & $\begin{array}{l}-124 \% \\
(p=0.889)\end{array}$ & $47 \%(p=0.012)$ \\
\hline $\begin{array}{l}130 \mathrm{keV} \\
\text { VMI+ O-MAR }\end{array}$ & $\begin{array}{l}81 \% \\
(p=0.001)\end{array}$ & $\begin{array}{l}83 \% \\
(p=0.001)\end{array}$ & $\begin{array}{l}66 \% \\
(p=0.001)\end{array}$ & $\begin{array}{l}91 \% \\
(p=0.001)\end{array}$ & $\begin{array}{l}79 \% \\
(p=0.012)\end{array}$ & $\begin{array}{l}46 \% \\
(p=0.012)\end{array}$ & $\begin{array}{l}32 \% \\
(p=0.012)\end{array}$ & $90 \%(p=0.012)$ \\
\hline
\end{tabular}

Table 4 Relative metal artifact reduction in CT values (HU), contrast-to-noise ratio, signal-to-noise ratio and noise in comparison to conventional (140kVp) with O-MAR images

\begin{tabular}{|c|c|c|c|c|c|c|c|c|}
\hline & \multicolumn{4}{|c|}{ Metal artifact reduction (\%) mildly affected pellets } & \multicolumn{4}{|c|}{ Metal artifact reduction (\%) severely affected pellets } \\
\hline & HU & CNR & SNR & Noise & HU & CNR & SNR & Noise \\
\hline 130 keV VMI & $\begin{array}{l}92 \% \\
(p=0.016)\end{array}$ & $\begin{array}{l}72 \% \\
(p=0.005)\end{array}$ & $\begin{array}{l}38 \% \\
(p=0.033)\end{array}$ & $\begin{array}{l}51 \% \\
(p=0.249)\end{array}$ & $\begin{array}{l}-331 \% \\
(p=0.025)\end{array}$ & $\begin{array}{l}-166 \% \\
(p=0.093)\end{array}$ & $\begin{array}{l}-202 \% \\
(p=0.050)\end{array}$ & $\begin{array}{l}-223 \% \\
(p=0.036)\end{array}$ \\
\hline $\begin{array}{l}130 \mathrm{keV} \\
\text { VMI + O-MAR }\end{array}$ & $\begin{array}{l}67 \% \\
(p=0.001)\end{array}$ & $\begin{array}{l}74 \% \\
(p=0.002)\end{array}$ & $\begin{array}{l}48 \% \\
(p=0.011)\end{array}$ & $\begin{array}{l}68 \% \\
(p=0.033)\end{array}$ & $6 \%(p=0.779)$ & $\begin{array}{l}15 \% \\
(p=0.208)\end{array}$ & $8 \%(p=0.208)$ & $39 \%(p=0.093)$ \\
\hline
\end{tabular}

These severe metal artifacts caused by bilateral implants were not reduced on $130 \mathrm{keV}$ VMI without O-MAR, which is supported by previous studies [6, $11,22]$. On the other hand, conventional imaging with O-MAR did result in a reduction of metal artifacts in severely affected pellets. Similarly, $130 \mathrm{keV}$ VMI with O-MAR resulted in a decrease of metal artifacts in severely affected pellets. In addition, $130 \mathrm{keV}$ VMI with O-MAR resulted in a decrease of metal artifacts in mildly affected pellets compared to conventional imaging with O-MAR and therefore resulted in the strongest metal artifact reduction for both mildly as severely affected pellets.

Our quantitative analysis using a THA phantom shows that high energy VMI with O-MAR results in the strongest metal artifact reduction, and this is supported by studies incorporating subjective assessment by radiologists [5-7] and by studies incorporating quantitative assessment of metal artifact reduction [5, $7,23]$. However, these studies used General Electric (GE) or Siemens systems to extract VMI with metal artifact reduction software, while we used a Philips system to extract VMI with O-MAR. Although metal artifact reduction techniques of the different vendors show comparable results regarding metal artifact reduction using VMI and VMI with metal artifact reduction software, it remains difficult to compare the results. A phantom study comparing metal artifact reduction of the different vendors would provide more insight in the performance of the different DECT techniques of the vendors and their metal artifact reduction software.

Although $130 \mathrm{keV}$ VMI with O-MAR resulted in the strongest relative metal artifact reduction, it should be noted that the CT values, CNR and SNR of the unaffected, mildly affected and severely affected pellets were lower in the $130 \mathrm{keV}$ VMI with O-MAR in comparison to conventional images with O-MAR. This can be explained by the high virtual monochromatic energy of the $130 \mathrm{keV}$ images, causing a reduction of the CT values in the images in comparison to conventional images. Although low SNR and CNR can affect the detectability of the pellets and therefore diligence is important, subjective analysis of patients shows that high energy VMI with O-MAR still can be assessed reliable [22]. Furthermore, the Rose criterion states that an object's CNR must exceed 3-5 to be detectable [24, 25]. Both conventional imaging with O-MAR as well as $130 \mathrm{keV}$ imaging with O-MAR exceeded this criterion for both mildly affected and severely affected pellets.

On our $130 \mathrm{keV}$ images, bright artifacts were induced. Likewise, several studies described that VMI and metal artifact reduction software can induce secondary artifacts $[7,26,27]$. Hence, the radiologist should also review conventional images in addition to the images with metal artifact reduction. Furthermore, relatively high negative metal artifact reduction in CNR $(-69 \%)$ and SNR $(-124 \%)$ was observed in severely affected pellets while high $p$ values ( 0.889 and 0.889 , respectively) were found 
when comparing $130 \mathrm{keV}$ VMI without O-MAR and conventional imaging without O-MAR, which seems contradictory. This may be explained by the large dispersion of CNR values and SNR values of the individual severely affected pellets in the $130 \mathrm{keV}$ VMI without O-MAR, caused by the introduction of bright artifacts.

In this study, we focused on image quality in orthopedic prosthesis imaging. Hence, we used a phantom with pellets mimicking bone tissue and we did not assess soft tissue. However, assessment of structures such as the bladder, uterus and prostate is also hampered by metal artifacts, which is particularly important in oncology patients. A study to quantify metal artifact reduction using structures mimicking soft tissue will certainly be of interest.

Our study has limitations. First, we categorized the pellets in the images with the unilateral and bilateral prostheses in unaffected, mildly affected and severely affected which allowed comprehensible analysis of all pellets. However, mildly affected pellets were caused by unilateral and bilateral prostheses. Assignment of these pellets to one category may not be in accordance with clinical practice because a unilateral prosthesis causes different artifacts than bilateral prostheses Furthermore, pellet L9 in the bilateral image was categorized as unaffected despite bright streaking artifacts, and a more appropriate category may be mildly affected instead. Third, our results did not quantify metal artifact reduction in the close vicinity of the implant. Fourth, the pellets only represent one bone density, comparable to trabecular bone. Bone structures with higher densities such as cortical bone may alter the results. Finally, we chose to place the background ROI in a part of the image where no metal artifacts were present. Although this avoids metal artifacts in the background ROI, the calculated CNR does not quantify contrast between pellets and the background in the vicinity of the pellets.

\section{Conclusions}

We conclude that $130 \mathrm{keV}$ VMI with O-MAR shows stronger metal artifact reduction over $130 \mathrm{keV}$ without O-MAR and conventional imaging with O-MAR. It should be encouraged to reconstruct high energy VMI with O-MAR besides conventional polychromatic images after THA.

\footnotetext{
Abbreviations

CNR: Contrast-to-noise ratio; CT: Computed tomography; DECT: Dual-energy computed tomography; HU: Hounsfield unit; IMR: Iterative model-based reconstruction; keV: Kiloelectronvolt; kVp: Kilovoltage peak; MAR: Metal artifact reduction; mGy: Milli-gray; O-MAR: Orthopedic metal artifact reduction; PMMA: Polymethyl methacrylate; ROI: Region of interest; SNR: Signal-to-noise ratio; THA: Total hip arthroplasty; TiAlV: Titanium-aluminum-vanadium;
}

UHMWPE: Ultrahigh molecular weight erthylene; VMI:Virtual monochromatic imaging; ZTA: Zirconia toughened aluminum.

\section{Supplementary Information}

The online version contains supplementary material available at https://doi. org/10.1186/s13244-021-01111-5.

Additional file 1. CT values, contrast-to-noise ratios, signal-to-noise ratios and noise values of all individual pellets for unilateral and bilateral protheses.

\section{Authors' contributions}

MS contributed to methodology, formal analysis, data curation, writing - original draft, writing - review and editing, and visualization. VHS contributed to conceptualization, investigation, data curation, writing-original draft, and project administration. RHHW contributed to methodology, conceptualization, and software. LvdR contributed to conceptualization, investigation, data curation, and project administration. IMN contributed to methodology, writing - original draft, and supervision. JACvO contributed to conceptualization and supervision. RWVH contributed to resources and investigation. TL contributed to resources and supervision. MFB contributed to conceptualization and supervision. All authors read and approved the final manuscript.

Funding

Not applicable.

Availability of data and materials

The datasets used and/or analyzed during the current study are available from the corresponding author on reasonable request.

\section{Declarations}

Ethics approval and consent to participate

Not applicable.

Consent for publication

Not applicable.

\section{Competing interests}

The authors declare that they have no competing interests.

\section{Author details}

${ }^{1}$ Department of Radiology, Isala, 8025 AB Zwolle, The Netherlands. ${ }^{2}$ Department of Radiology, Amsterdam University Medical Centre, 1105 AZ Amsterdam, The Netherlands. ${ }^{3}$ Department of Medical Physics, Isala, 8025 AB Zwolle, The Netherlands. ${ }^{4}$ Department of Radiology, University Medical Centre Utrecht, 3584 CX Utrecht, The Netherlands.

Received: 4 June 2021 Accepted: 18 October 2021

Published online: 24 November 2021

References

1. Wellenberg RHH, Boomsma MF, van Osch JAC et al (2017) Low-dose CT imaging of a total hip arthroplasty phantom using model-based iterative reconstruction and orthopedic metal artifact reduction. Skeletal Radiol. https://doi.org/10.1007/s00256-017-2580-2

2. Boomsma MF, Warringa N, Edens MA et al (2016) Quantitative analysis of orthopedic metal artefact reduction in 64-slice computed tomography scans in large head metal-on-metal total hip replacement, a phantom study. Springerplus. https://doi.org/10.1186/s40064-016-2006-y

3. Wellenberg RHH, Hakvoort ET, Slump CH, Boomsma MF, Maas M, Streekstra GJ (2018) Metal artifact reduction techniques in musculoskeletal CT-imaging. Eur J Radiol. https://doi.org/10.1016/j.ejrad.2018.08.010

4. Wellenberg RHH, Boomsma MF, van Osch JAC et al (2017) Quantifying metal artefact reduction using virtual monochromatic dual-layer detector 
spectral CT imaging in unilateral and bilateral total hip prostheses. Eur J Radiol 88:61-70. https://doi.org/10.1016/j.ejrad.2017.01.002

5. Bongers MN, Schabel C, Thomas C et al (2015) Comparison and combination of dual-energy- and iterative-based metal artefact reduction on hip prosthesis and dental implants. PLoS One. https://doi.org/10.1371/journ al.pone. 0143584

6. Andersson KM, Norrman E, Geijer H et al (2016) Visual grading evaluation of commercially available metal artefact reduction techniques in hip prosthesis computed tomography. Br J Radiol. https://doi.org/10.1259/ bjr.20150993

7. Han SC, Chung YE, Lee YH, Park KK, Kim MJ, Kim KW (2014) Metal artifact reduction software used with abdominopelvic dual-energy $\mathrm{CT}$ of patients with metal hip prostheses: assessment of image quality and clinical feasibility. AJR Am J Roentgenol. https://doi.org/10.2214/AJR.13. 10980

8. DeLee JG, Charnley J (1976) Radiological demarcation of cemented sockets in total hip replacement. Clin Orthop Relat Res. https://doi.org/ 10.1097/00003086-197611000-00003

9. Gruen T, McNeice G, Amstutz H (1979) Modes of failure of cemented stem-type femoral components: a radiographic analysis of loosening. Clin Orthop Relat Res 141:17-27

10. LROI. LROI Annual Report 2019. Dutch Athroplasty Regist. 2019

11. Wellenberg RHH, Boomsma MF, van Osch JAC et al (2017) Quantifying metal artefact reduction using virtual monochromatic dual-layer detector spectral CT imaging in unilateral and bilateral total hip prostheses. Eur J Radiol. https://doi.org/10.1016/j.ejrad.2017.01.002

12. Gjesteby L, De Man B, Jin Y et al (2016) Metal artifact reduction in CT: Where are we after four decades? IEEE Access. https://doi.org/10.1109/ ACCESS.2016.260862

13. Katsura M, Sato J, Akahane M, Kunimatsu A, Abe O (2018) Current and novel techniques for metal artifact reduction at CT: practical guide for radiologists. Radiographics. https://doi.org/10.1148/rg.2018170102

14. Lee M, Kim S, Lee S et al (2007) Overcoming artifacts from metallic orthopedic implants at high-field-strength MR imaging and multi-detector CT. Radiographics 27(3):791-803. https://doi.org/10.1148/RG.273065087

15. Lee IS, Kim HJ, Choi BK et al (2007) A pragmatic protocol for reduction in the metal artifact and radiation dose in multislice computed tomography of the spine: Cadaveric evaluation after cervical pedicle screw placement. J Comput Assist Tomogr. https://doi.org/10.1097/01.rct.0000250117. 18080.d8

16. Moon SG, Hong SH, Choi J-Y et al (2008) Metal artifact reduction by the alteration of technical factors in multidetector computed tomography. Comput Assist Tomogr 32(4):630-633. https://doi.org/10.1097/RCT.0b013 e3181568b27
17. Zhang K, Han Q, Xu X et al (2020) Metal artifact reduction of orthopedics metal artifact reduction algorithm in total hip and knee arthroplasty. Medicine (Baltimore). https://doi.org/10.1097/MD.0000000000019268

18. Wellenberg RHH, Boomsma MF, Van Osch JAC et al (2016) Computed tomography imaging of a hip prosthesis using iterativemodel-based reconstruction and orthopaedicmetal artefact reduction: a quantitative analysis. J Comput Assist Tomogr. https://doi.org/10.1097/RCT.00000 00000000449

19. Huang JY, Kerns JR, Nute JL et al (2015) An evaluation of three commercially available metal artifact reduction methods for $\mathrm{CT}$ imaging. Phys Med Biol. https://doi.org/10.1088/0031-9155/60/3/1047

20. Li H, Noel C, Chen H et al (2012) Clinical evaluation of a commercial orthopedic metal artifact reduction tool for $\mathrm{CT}$ simulations in radiation therapy. Med Phys. https://doi.org/10.1118/1.4762814

21. Hilgers G, Nuver T, Minken A (2014) The CT number accuracy of a novel commercial metal artifact reduction algorithm for large orthopedic implants. J Appl Clin Med Phys. https://doi.org/10.1120/jacmp.v15i1.4597

22. Neuhaus V, Grosse Hokamp N, Zopfs D et al (2019) Reducing artifacts from total hip replacements in dual layer detector CT: combination of virtual monoenergetic images and orthopedic metal artifact reduction. Eur J Radiol. https://doi.org/10.1016/j.ejrad.2018.12.008

23. Chou R, Chi HY, Lin YH, Ying LK, Chao YJ, Lin CH (2020) Comparison of quantitative measurements of four manufacturer's metal artifact reduction techniques for $\mathrm{CT}$ imaging with a self-made acrylic phantom. Technol Heal Care. https://doi.org/10.3233/THC-209028

24. Burgess AE (1999) The Rose model, revisited. J Opt Soc Am A. https://doi. org/10.1364/josaa.16.000633

25. Rose A (1973) Vision: human and electronic. Plenum, New York

26. Dabirrahmani D, Magnussen J, Appleyard RC (2015) Dual-energy computed tomography-How accurate is gemstone spectrum imaging metal artefact reduction? Its application to orthopedic metal implants. J Comput Assist Tomogr. https://doi.org/10.1097/RCT.0000000000000300

27. Pessis E, Sverzut JM, Campagna R, Guerini H, Feydy A, Drapé JL (2015) Reduction of metal artifact with dual-energy CT: virtual monospectral imaging with fast kilovoltage switching and metal artifact reduction software. Semin Musculoskelet Radiol. https://doi.org/10.1055/s-0035-15692 56

\section{Publisher's Note}

Springer Nature remains neutral with regard to jurisdictional claims in published maps and institutional affiliations.

\section{Submit your manuscript to a SpringerOpen ${ }^{\circ}$ journal and benefit from:}

- Convenient online submission

- Rigorous peer review

- Open access: articles freely available online

- High visibility within the field

- Retaining the copyright to your article

Submit your next manuscript at $\boldsymbol{\nabla}$ springeropen.com 